\title{
PROBLEMS OF INTERACTION OF VIBRATING SURFACES WITH PROCESSABLE MATERIALS
}

\author{
V. A. OSTAPENKO \\ Received 18 June 2004
}

We consider the mathematical model of interaction of a vibrating surface with the load placed on it. With the purpose of accounting for influence on behavior of not only system interaction of a blade with a load but also internal interaction of particles of a material, the load is submitted as a finite number of strips with zero thickness. The carrying blade is represented as a vibrating membrane. It is supposed that the weight of the material is comparable to or considerably surpasses the weight of the blade. Therefore, the model takes into account the inertia of the material. In the model with joint movement of the blade and the load, the separation opportunity of the load from the blade is provided. Therefore, there is a phase of separate movement of the blade and the load, with their subsequent connection accompanied with impact. The process of system movement is represented as alternating sequences of joint and separate movements of the load and the blade. The modeling of the process of the interaction of the load and the blade is represented as an initial-boundary value problem. The method of solution is developed and the exact solution of the set problem is obtained in a class of generalized functions.

\section{Introduction}

The experience of industrial application of various mechanisms using processing of materials on a vibrating surface has shown that the efficiency of such machines influences not only interaction of a material with a carrying surface, but also internal interaction of particles of a material. Therefore, for reception of additional parameters for optimization of work of such type of mechanisms, it is necessary to create a mathematical model allowing to dismember processable material on components, and then to provide each of such parts with the required characteristics.

\section{Statement of the problem}

With the purpose of the solution of such a problem, mathematical models are considered, in which the carrying blade is represented as an oscillating membrane or plate, and the processable material is represented as a series of strips. It was supposed that the weight of 
the material is comparable to or considerably surpasses the weight of the blade. Therefore, the model takes into account the inertia of the material. In case of a membrane, the movement of system is described by an equation of the kind

$$
u_{x x}+u_{y y}-\left(\frac{1}{a^{2}}+\sum_{i=1}^{n} \frac{\rho_{i}}{T} \delta\left(x-h_{i}\right)\right) u_{t t}=\sum_{i=1}^{n} \frac{g \rho_{i}}{T} \delta\left(x-h_{i}\right),
$$

where $\rho_{i}$ is $i$ 's layer of a material density, $\delta$-Dirac function, $h_{i}$-growing sequence of numbers. The oscillatory movements are imparted to the membrane at its edges, $T$ are the stretching efforts.

The carrying blade has a rectangular form with the sides of the rectangle parallel coordinate axes, and $0 \leq x \leq b, 0 \leq y \leq L$. To the sides $x=0$ and $x=b$ of this blade is applied harmonic moving; the parties $y=0$ and $y=L$ are free. It is assumed that on a blade along a straight line $x=h_{i}$, the thin layers of a load of density $\rho_{i}$ are located, the blade is stretched on its edges in a plane $x O y$ by efforts $T$.

In these assumptions, the problem is reduced to find in the domain $0<x<b, 0<y<L$, $t>0$ solution of (2.1), satisfying the boundary conditions

$$
\begin{aligned}
u(0, y, t)=A \sin \omega t, & u(b, y, t)=A \sin \omega t \\
u_{y}(x, 0, t)=0, & u_{y}(x, L, t)=0
\end{aligned}
$$

and the initial conditions

$$
u(x, y, 0)=0, \quad u_{t}(x, y, 0)=0, \quad 0<x<b, 0<y<L .
$$

\section{Solution of the first stage of the problem}

With the help of principle of superposition, the solution of the given problem is found as the sum of three functions

$$
u(x, y, t)=u_{1}(x, y, t)+u_{2}(x, y, t)+u_{3}(x, y, t) .
$$

Here the function $u_{1}(x, y, t)$ describes stationary oscillations of a blade with load and is represented as

$$
u_{1}(x, y, t)=U_{1}(x, y) \sin \omega t
$$

satisfying also the homogeneous equation appropriate (2.1), and the boundary conditions (2.2) and (2.3).

The function $u_{2}(x, y, t)$ describes free oscillations of a blade with a load arising by start from a status of equilibrium and rest, and is the solution of the homogeneous equation appropriate (2.1), satisfying the boundary conditions

$$
u(0, y, t)=0, \quad u(b, y, t)=0,
$$


the boundary conditions (2.3), and also the initial conditions

$$
u(x, y, 0)=-u_{1}(x, y, 0), \quad u_{t}(x, y, 0)=-u_{1, t}(x, y, 0) .
$$

At last, the function $u_{3}(x, y, t)$ describes oscillations of a blade arising owing to the sudden application of a load at $t=0$, and is the solution of (2.1) with the initial conditions (2.4) and the boundary conditions (2.3) and (3.3).

To find of all functions $u_{i}, i=1,2,3$, there comes up a question on obtaining the general solution of the equation

$$
X^{\prime \prime}(x)+\left[p+\sum_{i=1}^{n} q_{i}(x) \delta\left(x-h_{i}\right)\right] X(x)=\sum_{i=1}^{n} S_{i}(x) \delta\left(x-h_{i}\right) .
$$

Therefore, first of all, we will obtain the general solution of (3.5), using a method developed in [1].

The real general solution of the homogeneous equation

$$
X^{\prime \prime}(x)+p X(x)=0
$$

for $p<0$ is

$$
Z(x)=C_{1} \operatorname{sh} \sqrt{-p} x+C_{2} \operatorname{ch} \sqrt{-p} x,
$$

for $p>0$ is

$$
Z(x)=C_{1} \sin \sqrt{p} x+C_{2} \cos \sqrt{p} x,
$$

and for $p=0$ is $Z(x)=C_{1} x+C_{2}$.

Therefore, if $z_{i}(x)$ are the solutions of (3.6) which satisfy the initial conditions

$$
z_{i}\left(h_{i}\right)=0, \quad z_{i}^{\prime}\left(h_{i}\right)=1
$$

then for $p<0$,

$$
z_{i}(x)=\frac{1}{\sqrt{-p}} \operatorname{sh} \sqrt{-p}\left(x-h_{i}\right)
$$

for $p>0$,

$$
z_{i}(x)=\frac{1}{\sqrt{p}} \sin \sqrt{p}\left(x-h_{i}\right)
$$

and for $p=0, z_{i}(x)=x-h_{i}$.

Accordingly to [1], the general solution of (3.5) should be found as

$$
X(x)=Z(x)+\sum_{i=1}^{n} B_{i} z_{i}(x) H\left(x-h_{i}\right)
$$


where $H(x)$ is the Heavyside function. As a result of substitution of this form of the solution in (3.5), on account of the initial conditions (3.9), properties of $\delta$-function, and following from (3.12), equalities

$$
X\left(h_{1}\right)=Z\left(h_{1}\right), \quad X\left(h_{i}\right)=Z\left(h_{i}\right)+\sum_{j=1}^{i-1} B_{j} z_{j}\left(h_{i}\right),
$$

we obtain

$$
\begin{aligned}
& B_{1}=S_{1}\left(h_{1}\right)-q_{1}\left(h_{1}\right) Z\left(h_{1}\right), \\
& B_{i}=S_{i}\left(h_{i}\right)-q_{i}\left(h_{i}\right)\left[Z\left(h_{i}\right)+\sum_{j=1}^{i-1} B_{j} z_{j}\left(h_{i}\right)\right], \quad i=2, \ldots, n .
\end{aligned}
$$

Hence, the general solution of (3.5) is the function

$$
X(x)=Z(x)+\sum_{i=1}^{n}\left[S_{i}\left(h_{i}\right)-q_{i}\left(h_{i}\right)\left[Z\left(h_{i}\right)+\sum_{j=1}^{i-1} B_{j} z_{j}\left(h_{i}\right)\right]\right] z_{i}(x) H\left(x-h_{i}\right)
$$

Instead of recurrent formulas for factors $B_{i}$, it is more convenient to use obvious values of these factors,

$$
\begin{aligned}
B_{i}= & M_{i}-q_{i} \\
& \times \sum_{s(1)=1}^{i-1}\left[M_{s(1)} z_{s(1)}\left(h_{i}\right)-q_{s(1)} z_{s(1)}\left(h_{i}\right)\right. \\
& \times \sum_{s(2)=1}^{s(1)-1}\left[M_{s(2)} z_{s(2)}\left(h_{s(1)}\right)-q_{s(2)} z_{s(2)}\left(h_{s(1)}\right)\right. \\
& \times \sum_{s(3)=1}^{s(2)-1}\left[M_{s(3)} z_{s(3)}\left(h_{s(2)}\right)-q_{s(3)} z_{s(3)}\left(h_{s(2)}\right)\right. \\
& \times \sum_{s(3)-1}^{s(4)=1}\left[M_{s(4)} z_{s(4)}\left(h_{s(3)}\right)-q_{s(4)} z_{s(4)}\left(h_{s(3)}\right)\right. \\
& \times \sum_{s(5)=1}^{s(4)-1}\left[\ldots-q_{s(i-3)} z_{s(i-3)}\left(h_{s(i-4)}\right)\right. \\
& \times \sum_{s(i-3)-1}\left[M_{s(i-2)} z_{s(i-2)}\left(h_{s(i-3)}\right)-q_{s(i-2)} z_{s(i-2)}\left(h_{s(i-3)}\right)\right. \\
& \left.\left.\left.\left.\left.\times \sum_{s(i-2)=1}^{\sum_{s(i-2)-1}} M_{s(i-1)} z_{s(i-1)}\left(h_{s(i-2)}\right)\right] \ldots\right]\right]\right]\right]
\end{aligned}
$$


which are obtained as a result of the solutions of recurrent equalities. Here

$$
M_{1}=B_{1}, \quad M_{i}=S_{i}\left(h_{i}\right)-q_{i}\left(h_{i}\right) Z\left(h_{i}\right), \quad i=2, \ldots, n .
$$

With the purpose of reception of the decision in an obvious and most evident kind, we will state in this paper the solution of the problem for a case of two symmetrically located strips of a load. We assume $n=2, x_{1}=h, x_{2}=b-h$, and $0<h<b / 2$.

In this special case the function $X(x)$ is represented as follows. For $p<0$,

$$
\begin{aligned}
& X(x)=C_{1} \operatorname{sh} \sqrt{-p} x+C_{2} \operatorname{ch} \sqrt{-p} x \\
&+\left[S_{1}(h)-q_{1}(h)\left(C_{1} \operatorname{sh} \sqrt{-p} h+C_{2} \operatorname{ch} \sqrt{-p} h\right)\right] \operatorname{sh} \sqrt{-p}(x-h) H(x-h) \times \frac{1}{\sqrt{-p}} \\
&+\left\{S_{2}(b-h)-q_{2}(b-h)[\right. C_{1} \operatorname{sh} \sqrt{-p}(b-h)+C_{2} \operatorname{ch} \sqrt{-p}(b-h) \\
&+\left[S_{1}(h)-q_{1}(h)\left(C_{1} \operatorname{sh} \sqrt{-p} h+C_{2} \operatorname{ch} \sqrt{-p} h\right)\right] \\
&\left.\left.\times \operatorname{sh} \sqrt{-p}(b-2 h) \times \frac{1}{\sqrt{-p}}\right]\right\}
\end{aligned}
$$

For $p>0$,

$$
\begin{aligned}
& X(x)=C_{1} \sin \sqrt{p} x+C_{2} \cos \sqrt{p} x \\
&+\left[S_{1}(h)-q_{1}(h)\left(C_{1} \sin \sqrt{p} h+C_{2} \cos \sqrt{p} h\right)\right] \sin \sqrt{p}(x-h) H(x-h) \times \frac{1}{\sqrt{p}} \\
&+\left\{S_{2}(b-h)-q_{2}(b-h)[\right. {\left[C_{1} \sin \sqrt{p}(b-h)+C_{2} \cos \sqrt{p}(b-h)\right.} \\
&+ {\left[S_{1}(h)-q_{1}(h)\left(C_{1} \sin \sqrt{p} h+C_{2} \cos \sqrt{p} h\right)\right] } \\
&\left.\left.\times \sin \sqrt{p}(b-2 h) \times \frac{1}{\sqrt{p}}\right]\right\} \\
& \times \sin \sqrt{p}(x-(b-h)) H(x-(b-h)) \frac{1}{\sqrt{p}} .
\end{aligned}
$$

For $p=0$,

$$
\begin{aligned}
X(x)= & C_{1} x+C_{2}\left[S_{1}(h)-q_{1}(h)\left(C_{1} h+C_{2}\right)\right](x-h) H(x-h) \\
& +\left\{S_{2}(b-h)-q_{2}(b-h)\left[C_{1}(b-h)+C_{2}+\left[S_{1}(h)-q_{1}(h)\left(C_{1} h-C_{2}\right)\right](b-2 h)\right]\right\} \\
& \times(x-(b-h)) H(x-(b-h)) .
\end{aligned}
$$


We consider the problem of obtaining a function $u_{1}(x, y, t)$. Substituting the form of the solution (3.2) in the homogeneous equation (2.1), we obtain the equation for the function $U_{1}(x, y)$ :

$$
U_{1, x x}(x, y)+U_{1, y y}(x, y)+\omega^{2}\left(\frac{1}{a^{2}}+\frac{\rho_{H}}{T}(\delta(x-h)+\delta(x-(b-h)))\right)+U_{1}(x, y)=0 .
$$

To solve (3.21) we will apply a method of separation variables in the form

$$
U_{1}(x, y)=X(x) Y(y) .
$$

As a result of separation variables, we obtain system of the two equations

$$
\begin{gathered}
X^{\prime \prime}+\left[\omega^{2}\left(\frac{1}{a^{2}}+\frac{\rho_{H}}{T}(\delta(x-h)+\delta(x-(b-h)))\right)-\lambda\right] X=0, \\
Y^{\prime \prime}+\lambda Y=0,
\end{gathered}
$$

with the boundary conditions

$$
\begin{array}{rlrl}
X(0) Y(y) & =A, & & X(b) Y(y)=A, \\
Y^{\prime}(0) & =0, & Y^{\prime}(L)=0 .
\end{array}
$$

From (3.24) it follows that

$$
Y(y)=\text { const. }
$$

The second equation (3.23) has a solution of the kind (3.26) only for $\lambda=0$. Thus, the only eigenvalue of a problem (3.23), (3.25) under condition (3.26) is the number $\lambda=0$. Without loss in generality, we accept $Y(y)=1$.

For $\lambda=0$, general solution of the first equation (3.23) turns out from the formula (3.19) at

$$
q_{1}=q_{2}=\frac{\rho_{H} \omega^{2}}{T}, \quad S_{1}=S_{2}=0, \quad p=\frac{\omega^{2}}{a^{2}},
$$

and consequently it looks as

$$
\begin{aligned}
X(x)= & C_{1} \sin \frac{\omega}{a} x \\
& +C_{2} \cos \frac{\omega}{a} x-\frac{\rho_{H} \omega^{2}}{T}\left(C_{1} \sin \frac{\omega}{a} h+C_{2} \cos \frac{\omega}{a} h\right) \frac{a}{\omega} \sin \frac{\omega}{a}(x-h) H(x-h) \\
- & \frac{\rho_{H} \omega^{2}}{T}\left\{C_{1} \sin \frac{\omega}{a}(b-h)+C_{2} \cos \frac{\omega}{a}(b-h)\right. \\
& \left.\quad-\frac{\rho_{H} \omega^{2}}{T}\left(C_{1} \sin \frac{\omega}{a} h+C_{2} \cos \frac{\omega}{a} h\right) \frac{a}{\omega} \sin \frac{\omega}{a}(b-2 h)\right\} \frac{a}{\omega} \\
& \times \sin \frac{\omega}{a}(x-(b-h)) H(x-(b-h)) .
\end{aligned}
$$


From (3.24) we obtain $X(0)=A, X(b)=A$, whence and from (3.28) we determine constants of integration. Thus, having designated

$$
D=\sin \frac{\omega}{a} b-2 \frac{a \omega \rho_{H}}{T} \sin \frac{\omega}{a} h \sin \frac{\omega}{a}(b-h)+\left(\frac{a \omega \rho_{H}}{T}\right)^{2} \sin ^{2} \frac{\omega}{a} h \sin \frac{\omega}{a}(b-2 h),
$$

we will have

$$
\begin{aligned}
X(x)=\frac{A}{D}\{ & \sin \frac{\omega}{a} x+\sin \frac{\omega}{a}(b-x)+\frac{a \omega \rho_{H}}{T}\left(\cos \frac{\omega}{a}(b-x)-\cos \frac{\omega}{a}(b-2 h) \cos \frac{\omega}{a} x\right) \\
- & \left(\frac{a \omega \rho_{H}}{T}\right)^{2} \sin \frac{\omega}{a} h \sin \frac{\omega}{a}(b-2 h) \sin \frac{\omega}{a}(x-h) \\
- & \frac{a \omega \rho_{H}}{T}\left[\sin \frac{\omega}{a} h+\sin \frac{\omega}{a}(b-h)-\frac{a \omega \rho_{H}}{T} \sin \frac{\omega}{a} h \sin \frac{\omega}{a}(b-2 h)\right] \\
\times & \sin \frac{\omega}{a}(x-h) H(x-h) \\
- & \frac{a \omega \rho_{H}}{T}\left[\sin \frac{\omega}{a} h+\sin \frac{\omega}{a}(b-h)+\frac{a \omega \rho_{H}}{T} \sin \frac{\omega}{a}(b-h) \sin \frac{\omega}{a}(b-2 h)\right. \\
& \quad-\left(\frac{a \omega \rho_{H}}{T}\right)^{2} \sin \frac{\omega}{a} h \sin \frac{\omega}{a}(b-2 h) \\
& \quad-\frac{a \omega \rho_{H}}{T}\left[\sin \frac{\omega}{a} h+\sin \frac{\omega}{a}(b-h)-\frac{a \omega \rho_{H}}{T} \sin \frac{\omega}{a} h \sin \frac{\omega}{a}(b-2 h)\right] \\
& \left.\left.\times \sin \frac{\omega}{a}(b-2 h)\right] \sin \frac{\omega}{a}(x-(b-h)) H(x-(b-h))\right\} .
\end{aligned}
$$

Thus, the solution of a problem for function $u_{1}$ looks as

$$
u_{1}(x, y, t)=X(x) \sin \omega t
$$

where $X(x)$ is determined by formula (3.30), and $D$ by formula (3.29).

We consider now problem on finding function $u_{2}(x, y, t)$. Separating variables, we will find function $u_{2}(x, y, t)$ as

$$
u_{2}(x, y, t)=U_{2}(x, y) T(t)
$$

The substitution of the form of the solution (3.32) in the homogeneous equation (2.1) results in the two equations

$$
\begin{gathered}
U_{2, x x}+U_{2, y y}+\lambda\left(\frac{1}{a^{2}}+\frac{\rho_{H}}{T} \delta(x-h)+\frac{\rho_{H}}{T} \delta(x-(b-h))\right) U_{2}=0, \\
T^{\prime \prime}+\lambda T=0 .
\end{gathered}
$$

Solution of the first equation in (3.33) also is found by method of separation variables as

$$
U_{2}(x, y)=X(x) Y(y)
$$


In result there appears a boundary problem of Sturm and Liouville about integration of system of the equations

$$
\begin{gathered}
Y^{\prime \prime}+\mu Y=0, \\
X^{\prime \prime}+\left[\lambda\left(\frac{1}{a^{2}}+\frac{\rho_{H}}{T} \delta(x-h)+\frac{\rho_{H}}{T} \delta(x-(b-h))\right)-\mu\right] X=0,
\end{gathered}
$$

with boundary conditions

$$
\begin{aligned}
Y^{\prime}(0) & =0, & & Y^{\prime}(L)=0, \\
X(0) & =0, & & X(b)=0 .
\end{aligned}
$$

It is known that the problem of Sturm and Liouville for the first equation in (3.35) with boundary conditions (3.36) has eigenvalues

$$
\mu_{n}=\left(\frac{\pi n}{L}\right)^{2}, \quad n=0,1,2, \ldots
$$

and the eigenfunctions

$$
Y_{n}(y)=C_{n} \cos \frac{\pi n}{L} y, \quad n=0,1,2, \ldots
$$

The second equation in (3.35) at $n=2, S_{1}=S_{2}=0, p=\left(\lambda / a^{2}\right)-\mu_{n}, q_{1}=q_{2}=\left(\lambda \rho_{H} / T\right)$ coincides with (3.5).

We consider a problem of Sturm and Liouville for the second equation in (3.35) with boundary conditions (3.37). For this purpose it is necessary to consider three variants separately.

(a) $p<0$, or $\lambda<(\pi n a / L)^{2}, n=0,1,2 \ldots$ In this case, general solution of the second equation in (3.35) looks as (3.18), and on account of values $\lambda=a^{2}\left(p+\mu_{n}\right), q_{1}=q_{2}=$ $(1 / T) a^{2} \rho_{H}\left(p+\mu_{n}\right)$, this general solution can be written down as

$$
\begin{aligned}
X(x)=C_{1} \operatorname{sh} \sqrt{-p} x+C_{2} c h \sqrt{-p} x & \\
-\frac{1}{T} a^{2} \rho_{H}\left(p+\mu_{n}\right) & \left(C_{1} s h \sqrt{-p} h+C_{2} \frac{1}{\sqrt{-p}} c h \sqrt{-p} h\right) \operatorname{sh} \sqrt{-p}(x-h) H(x-h) \\
-\frac{1}{T} a^{2} \rho_{H}\left(p+\mu_{n}\right)[ & {\left[C_{1} s h \sqrt{-p}(b-h)+C_{2} c h \sqrt{-p}(b-h)\right.} \\
& -\frac{1}{T} a^{2} \rho_{H}\left(p+\mu_{n}\right)\left(C_{1} \operatorname{sh} \sqrt{-p} h+C_{2} c h \sqrt{-p} h\right) \operatorname{sh} \sqrt{-p}(b-2 h) \\
& \left.\times \frac{1}{\sqrt{-p}}\right] \operatorname{sh} \sqrt{-p}(x-(b-h)) \times \frac{1}{\sqrt{-p}} H(x-(b-h)) .
\end{aligned}
$$


As a result of substitution of this general solution in boundary conditions (3.37), two equalities $C_{2}=0, C_{1} G(z)=0$ turn out, where $z=\sqrt{-p} b, \alpha=h / b, \gamma=(1 / T) a^{2} \rho_{H}$,

$$
\begin{aligned}
G(z)= & \operatorname{sh} z-2 \frac{\gamma}{b z}\left(b^{2} \mu_{n}-z^{2}\right) \operatorname{sh} \alpha z \operatorname{sh}(1-\alpha) z \\
& +\left(\frac{\gamma}{b}\left(b^{2} \mu_{n}-z^{2}\right)\right)^{2} \operatorname{sh}^{2} \alpha z \operatorname{sh} z(1-2 \alpha) .
\end{aligned}
$$

Therefore the question on existence of eigenvalues of a considered boundary problem for $p<0$ is reduced to a question on existence of positive roots of function $G(z)$. In [3], it is shown that at $1 / \alpha<\gamma \mu_{n} b<1 /(\alpha(1-\alpha))$ such positive roots $z_{n}$ exist. In this case boundary problem for $X(x)$ has the eigenvalues

$$
\lambda_{n}=a^{2}\left(\mu_{n}-\left(\frac{z_{n}}{b}\right)^{2}\right)
$$

and the eigenfunctions

$$
\begin{aligned}
X(x)=C_{n}[ & \operatorname{sh} \frac{z_{n}}{b} x-a^{2} \frac{\rho_{H}}{T b z_{n}}\left(b^{2} \mu_{n}-z_{n}^{2}\right) \operatorname{sh} \frac{z_{n}}{b} h \operatorname{sh} \frac{z_{n}}{b}(x-h) H(x-h)-a^{2} \frac{\rho_{H}}{T b z_{n}}\left(b^{2} \mu_{n}-z_{n}^{2}\right) \\
& \times\left[\operatorname{sh} \frac{z_{n}}{b}(b-h)-a^{2} \frac{\rho_{H}}{T b z_{n}}\left(b^{2} \mu_{n}-z_{n}^{2}\right) \operatorname{sh} \frac{z_{n}}{b} h \operatorname{sh} \frac{z_{n}}{b}(b-2 h)\right] \\
& \left.\times \operatorname{sh} \frac{z_{n}}{b}(x-(b-h)) H(x-(b-h))\right] .
\end{aligned}
$$

(b) $p=0$, that is, $\lambda_{n}=(\pi n(a / L))^{2}, n=0,1,2, \ldots$ Denoting $q=(1 / T)(\pi n(a / L))^{2} \rho_{H}$, we will have in this case that the general solution of the second equation in (3.35) looks as (3.20) at $S_{1}=S_{2}=0, q_{1}(h)=q_{2}(h)=q$. The substitution of this solution in boundary conditions (3.37) gives

$$
\begin{gathered}
C_{2}=0, \\
C_{1}\left[b-2 q h(b-h)+q^{2} h^{2}(b-2 h)\right]=0 .
\end{gathered}
$$

Roots of function in square brackets are $q_{1}=b / h(b-2 h)$ and $q_{2}=1 / h$, whence

$$
n_{1}=\frac{L}{\pi a} \sqrt{\frac{T b}{\rho_{H} h(b-2 h)}} ; \quad n_{2}=\frac{L}{\pi a} \sqrt{\frac{T}{\rho_{H} h}}
$$

Generally, numbers $n_{1}$ and $n_{2}$ will not be natural, and it will mean that for $p=0$ the boundary problem (3.35), (3.37) has no eigenvalues. But, if one of numbers $n_{1}$ or $n_{2}$, or both these numbers are integers, the boundary problem (3.35), (3.37) will have one or 
two eigenvalues $\lambda_{n 1}=\left(\pi n_{1}(a / L)\right)^{2}$ and $\lambda_{n 2}=\left(\pi n_{2}(a / L)\right)^{2}$ and one or two eigenfunctions

$$
\begin{aligned}
X_{n i}(x)=C_{n i}[ & x-\frac{\rho_{H}}{T}\left(\pi n_{i} \frac{a}{L}\right)^{2} h(x-h) H(x-h) \\
& -\frac{\rho_{H}}{T}\left(\pi n_{i} \frac{a}{L}\right)^{2}\left[(b-h)-\frac{\rho_{H}}{T}\left(\pi n_{i} \frac{a}{L}\right)^{2} h(b-2 h)\right] \\
& \times(x-(b-h)) H(x-(b-h))] .
\end{aligned}
$$

(c) $p>0$, that is, $\lambda>(\pi n(a / L))^{2}, n=0,1,2, \ldots$ In this case general solution of the second equation in (3.35) looks as (3.19) at $S_{1}=S_{2}=0, q_{1}(h)=q_{2}(b-h)=a^{2}\left(\rho_{H} / T\right)\left(\mu_{n}+p\right)$, $\lambda=a^{2}\left(\mu_{n}+p\right)$.

Therefore, substituting (3.19) in boundary conditions (3.37), we will have $C_{2}=0$, $C_{1} G(z)=0$, where we denote $z=\sqrt{p} b, \alpha=h / b$,

$$
\begin{aligned}
G(z)= & \sin z-2 a^{2} \frac{\rho_{H}}{T b z}\left(b^{2} \mu_{n}+z^{2}\right) \sin \alpha z \sin (1-\alpha) z \\
& +\left(a^{2} \frac{\rho_{H}}{T b}\left(b^{2} \mu_{n}+z^{2}\right) \frac{1}{z}\right)^{2} \sin ^{2} \alpha z \sin (1-2 \alpha) z .
\end{aligned}
$$

In [3], it is shown that the function $G(z)$ has at each $n$ a countable set of positive roots in points $z_{n m}, n=0,1,2, \ldots, m=1,2, \ldots$. Here roots $z_{n m}$ are supplied with an index $m$ in order of their increase. Then $\left(p_{n m}\right)^{1 / 2}=\left(z_{n m} / b\right), \lambda_{n m}=a^{2}\left(\left(z_{n m} / b\right)^{2}+(\pi(n / L))^{2}\right)$, $q_{n m}=a^{2}\left(\rho_{H} / T\right)\left(\left(z_{n m} / b\right)^{2}+(\pi(n / L))^{2}\right), n, m=0,1,2, \ldots$

The eigenfunctions of a boundary problem for the second equation (3.35) with boundary conditions (3.37), corresponding to eigenvalues $\lambda_{n m}$, look as

$$
\begin{aligned}
& X_{n m}(x) \\
& =C_{n m}\left[\sin z_{n m} \frac{x}{b}-a^{2} \frac{\rho_{H}}{T b}\left(\left(\pi b \frac{n}{L}\right)^{2}+z_{n m}^{2}\right) \frac{1}{z_{n m}}\right. \\
& \times \sin z_{n m} \frac{1}{b} h \sin z_{n m} \frac{1}{b}(x-h) H(x-h)-a^{2} \frac{\rho_{H}}{T b}\left(\left(\pi b \frac{n}{L}\right)^{2}+z_{n m}^{2}\right) \frac{1}{z_{n m}} \\
& \times\left[\sin z_{n m} \frac{1}{b}(b-h)-a^{2} \frac{\rho_{H}}{T b}\left(\left(\pi b \frac{n}{L}\right)^{2}+z_{n m}^{2}\right) \frac{1}{z_{n m}} \sin z_{n m} \frac{1}{b} h\right. \\
& \left.\left.\times \sin z_{n m} \frac{1}{b}(b-2 h)\right] \sin z_{n m} \frac{1}{b}(x-(b-h)) H(x-(b-h))\right] .
\end{aligned}
$$

The functions $T(t)$ now are easily determined. 
Hence, solution $u_{2}$ can be written as

$$
\begin{aligned}
& u_{2}(x, y, t) \\
& =\sum_{n=0}^{\infty} \sum_{m=1}^{\infty} \cos \pi y \frac{n}{L}\left[\sin \frac{z_{n m}}{b} x-a^{2} \frac{\rho_{H}}{T b}\left(\left(\pi b \frac{n}{L}\right)^{2}+z_{n m}^{2}\right) \frac{1}{z_{n m}} \sin \frac{z_{n m}}{b} h\right. \\
& \times \sin \frac{z_{n m}}{b}(x-h) H(x-h)-a^{2} \frac{\rho_{H}}{T b}\left(\left(\pi b \frac{n}{L}\right)^{2}+z_{n m}^{2}\right) \frac{1}{z_{n m}} \\
& \times\left[\sin \frac{z_{n m}}{b}(b-h)-a^{2} \frac{\rho_{H}}{T b}\left(\left(\pi b \frac{n}{L}\right)^{2}+z_{n m}^{2}\right) \frac{1}{z_{n m}}\right. \\
& \left.\times \sin \frac{z_{n m}}{b} h \sin \frac{z_{n m}}{b}(b-2 h)\right] \\
& \left.\times \sin \frac{z_{n m}}{b}(x-(b-h)) H(x-(b-h))\right] \\
& \times\left[A_{n m} \sin \frac{a}{b}\left(\left(\pi b \frac{n}{L}\right)^{2}+z_{n m}^{2}\right)^{1 / 2} t+B_{n m} \cos \frac{a}{b}\left(\left(\pi b \frac{n}{L}\right)^{2}+z_{n m}^{2}\right)^{1 / 2} t\right] \\
& +\sum_{n>n 2}^{n<n 1} \cos \pi y \frac{n}{L}\left[\operatorname{sh} \frac{z_{n}}{b} x-a^{2} \frac{\rho_{H}}{T b}\left(\left(\pi b \frac{n}{L}\right)^{2}-z_{n}^{2}\right)\right. \\
& \times \frac{1}{z_{n}} \operatorname{sh} \frac{z_{n}}{b} h \operatorname{sh} \frac{z_{n}}{b}(x-h) H(x-h)-a^{2} \frac{\rho_{H}}{T b}\left(\left(\pi b \frac{n}{L}\right)^{2}-z_{n}^{2}\right) \frac{1}{z_{n}} \\
& \times\left[\operatorname{sh} \frac{z_{n}}{b}(b-h)-a^{2} \frac{\rho_{H}}{T b}\left(\left(\pi b \frac{n}{L}\right)^{2}-z_{n}^{2}\right) \frac{1}{z_{n}} \operatorname{sh} \frac{z_{n}}{b} h \operatorname{sh} \frac{z_{n}}{b}(b-2 h)\right] \\
& \left.\times \operatorname{sh} \frac{z_{n}}{b}(x-(b-h)) \times H(x-(b-h))\right] \\
& \times\left[A_{n} \sin \frac{a}{b}\left(\left(\pi b \frac{n}{L}\right)^{2}-z_{n}^{2}\right)^{1 / 2} t+B_{n} \cos \frac{a}{b}\left(\left(\pi b \frac{n}{L}\right)^{2}-z_{n}^{2}\right)^{1 / 2} t\right] \\
& +\sum_{i=1}^{2} \cos \pi y \frac{n}{L}\left[x-\frac{\rho_{H}}{T}\left(\pi n_{i} \frac{a}{L}\right)^{2} h(x-h) H(x-h)-\frac{\rho_{H}}{T}\left(\pi n_{i} \frac{a}{L}\right)^{2}\right. \\
& \left.\times\left[(b-h)-\frac{\rho_{H}}{T}\left(\pi n_{i} \frac{a}{L}\right)^{2} h(b-2 h)\right](x-(b-h)) H(x-(b-h))\right] \\
& \times\left[A_{n i} \sin \pi n_{i} \frac{a}{L} t+B_{n i} \cos \pi n_{i} \frac{a}{L} t\right]
\end{aligned}
$$

with arbitrary constants $A_{n m}, B_{n m}, A_{n}, B_{n}, A_{n i}, B_{n i}$. In the general case for obtaining these constants, it is necessary to expand the right parts of the initial conditions (3.4) as

$$
-u_{1}(x, y, 0)=\sum_{n=0}^{\infty} \varphi_{n}(x) \cos \pi y \frac{n}{L}, \quad-u_{1, t}(x, y, 0)=\sum_{n=0}^{\infty} \psi_{n}(x) \cos \pi y \frac{n}{L},
$$


where

$$
\varphi_{n}(x)=\sum_{m=1}^{\infty} b_{n m} X_{n m}(x), \quad \psi_{n}(x)=\sum_{m=1}^{\infty} c_{n m} X_{n m}(x) .
$$

It can be shown that the eigenfunctions $X n m(x)$, responding to various eigenvalues $\lambda_{n m}$, are linearly independent, but not orthogonal on an interval $[0, b]$. Therefore coefficients of expansion $b_{n m}$ and $c_{n m}$ have to be determined from infinite system of the linear equations

$$
\begin{gathered}
\sum_{m=1}^{\infty} b_{n m} \int_{0}^{b} X_{n m}(x) X_{n s}(x) d x=\int_{0}^{b} \varphi_{n}(x) X_{n m}(x) d x \\
\sum_{m=1}^{\infty} c_{n m} \int_{0}^{b} X_{n m}(x) X_{n s}(x) d x=\int_{0}^{b} \psi_{n}(x) X_{n m}(x) d x, \quad s=1,2, \ldots ; n=0,1,2, \ldots
\end{gathered}
$$

Determinants of these systems are determinants of Gramme, therefore these systems have unique solution for $b_{n m}$ and $c_{n m}$, respectively.

In a considered special case the kind of the solution $u_{1}$ permits the formula (3.49) to be limited only to double sum, and therefore

$$
B_{n m}=b_{n m}, \quad A_{n m}=\frac{b c_{n m}}{a\left((\pi b n / L)^{2}+z_{n m}^{2}\right)^{1 / 2}} .
$$

Moreover, in this case only $\mathcal{c}_{0 m}$ and hence $A_{0 m}$ will be distinct from zero. Therefore, final kind of function $u_{2}$ becomes essentially simpler.

We will turn now to a question on finding function $u_{3}(x, y, t)$, which we will search as the sum of two functions:

$$
u_{3}(x, y, t)=u_{31}(x, y, t)+u_{32}(x, y, t)
$$

where $u_{31}(x, y, t)$ is static deflection of a membrane

$$
u_{31}(x)=\rho_{H} \frac{g}{T}(-x+(x-h) H(x-h)+(x-(b-h)) H(x-(b-h))),
$$

and $u_{32}(x, y, t)$ is the solution of the homogeneous equation (2.1), satisfying the boundary conditions (2.3) and (3.3) and providing for function $u_{3}(x, y, t)$ satisfaction to the initial conditions (2.4).

Now initial conditions for function $u_{32}$ will be

$$
u_{32}(x, y, 0)=-u_{31}(x), \quad u_{32, t}(x, y, 0)=0 .
$$

The obtaining of function $u_{32}$ also is carried out by a method of separation variables, for which it is sufficient to use the form of the solution (3.49). Taking into account a kind of the initial conditions (3.56), in formula (3.49) distinct from zero will be only coefficients $B_{0 m}$, determined from (3.53), and constants $b_{0 m}$ will be determined from the first system (3.52), in which it is necessary to put $\varphi_{0}(x)=-u_{31}(x)$. 
So,

$$
\begin{aligned}
& u_{32}(x, y, t) \\
& \begin{aligned}
=\sum_{m=1}^{\infty} B_{0 m} \cos a \frac{z_{0 m}}{b} t[ & \sin \frac{z_{0 m}}{b} x-a^{2} \frac{\rho_{H}}{T b} z_{0 m} \sin \frac{z_{0 m}}{b} h \sin \frac{z_{0 m}}{b}(x-h) H(x-h) \\
-a^{2} \frac{\rho_{H}}{T b} z_{0 m} & {\left[\sin \frac{z_{0 m}}{b}(b-h)-a^{2} \frac{\rho_{H}}{T b} z_{0 m} \sin \frac{z_{0 m}}{b} h\right.} \\
& \left.\left.\times \sin \frac{z_{0 m}}{b}(b-2 h)\right] \sin \frac{z_{0 m}}{b}(x-(b-h)) H(x-(b-h))\right] .
\end{aligned}
\end{aligned}
$$

Thus, the solution of a problem on the movement of a blade and a load without separation is obtained.

\section{The separate movement of load and blade}

At large enough acceleration the separation of a load from a blade can take place. The necessary and sufficient conditions for separation look as

$$
u_{t t}\left(h_{i}, t\right)=-g, \quad u_{t t t}\left(h_{i}, t_{\text {OTP }}\right)<0,
$$

where $t_{\text {OTP }}$ is the least positive root of the first equation (4.1), determining the moment of possible separation of a load. If the separation will take place, the structure of system

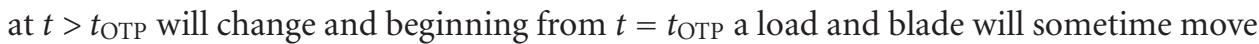
separately. The movement of a blade will be determined by function $v(x, t)$, being on an interval $0<x<b$ and at $t>t_{\text {OTP }}$ by the solution of the wave equation

$$
v_{x x}-\frac{1}{a^{2}} v_{t t}=0,
$$

satisfying the initial conditions

$$
v\left(x, t_{\mathrm{OTP}}\right)=u\left(x, t_{\mathrm{OTP}}\right), \quad v_{t}\left(x, t_{\mathrm{OTP}}\right)=u_{t}\left(x, t_{\mathrm{OTP}}\right),
$$

and the boundary conditions

$$
v(0, t)=A \sin \omega t \quad v(b, t)=A \sin \omega t .
$$

The movement of a load will be described by the equation

$$
z(t)=-\frac{g}{2}\left(t-t_{\mathrm{OTP}}\right)^{2}+u_{t}\left(h, t_{\mathrm{OTP}}\right)\left(t-t_{\mathrm{OTP}}\right)+u\left(h, t_{\mathrm{OTP}}\right) .
$$

Solution of the problem (4.2), (4.3), (4.4) is represented as

$$
v(x, t)=v_{1}(x, t)+v_{2}(x, t),
$$

where the function $v_{1}(x, t)$ looks like

$$
v_{1}(x, t)=X_{1}(x) \sin \omega t
$$

satisfying (4.2) and boundary conditions (4.4). 
The substitution (4.7) in (4.2) results in the equation

$$
X_{1}^{\prime \prime}(x)+\frac{\omega^{2}}{a^{2}} X_{1}(x)=0,
$$

whose general solution is the function

$$
X_{1}(x)=B \sin \frac{x \omega}{a}+C \cos \frac{x \omega}{a} .
$$

Substituting (4.7) in boundary conditions (4.4) and taking into account (4.9), we will receive system of the equations

$$
C=A, \quad B \sin \frac{b \omega}{a}+C \cos \frac{b \omega}{a}=A,
$$

from which are obtained

$$
B=\frac{A}{\sin (b \omega / a)}\left(1-\cos \frac{b \omega}{a}\right), \quad C=A .
$$

Therefore,

$$
X_{1}(x)=\frac{A}{\sin (b \omega / a)}\left(\sin \frac{x \omega}{a}+\sin \frac{(b-x) \omega}{a}\right) .
$$

Then the function $v_{2}(x, t)$ should be the solution of (4.2), satisfying the homogeneous boundary conditions

$$
v_{2}(0, t)=0, \quad v_{2}(b, t)=0,
$$

and the initial conditions

$$
\begin{aligned}
v_{2}\left(x, t_{\text {OTP }}\right) & =u\left(x, t_{\text {OTP }}\right)-X_{1}(x) \sin \omega t_{\text {OTP }}=\varphi(x), \\
v_{2, t}\left(x, t_{\text {OTP }}\right) & =u_{t}\left(x, t_{\text {OTP }}\right)-\omega X_{1}(x) \sin \omega t_{\text {OTP }}=\psi(x) .
\end{aligned}
$$

The problem of searching for the function $v_{2}(x, t)$ can be solved by the method of continuations or Fourier method. In case of application of the Fourier method, the function $v_{2}(x, t)$ will look like [4, pages $\left.82-85\right]$

$$
v_{2}(x, t)=\sum_{n=1}^{\infty}\left(A_{n} \sin \frac{\pi n a t}{b}+B_{n} \cos \frac{\pi n a t}{b}\right) \sin \frac{\pi n x}{b},
$$

and the factors $A_{n}$ and $B_{n}$ are determined from equalities

$$
\begin{gathered}
A_{n} \sin \frac{\pi n a t_{o m p}}{b}+B_{n} \cos \frac{\pi n a t_{o m p}}{b}=N_{n}, \\
A_{n} \sin \frac{\pi n a t_{o m p}}{b}+B_{n} \cos \frac{\pi n a t_{o m p}}{b}=\frac{b K_{n}}{\pi n a},
\end{gathered}
$$


that is,

$$
\begin{aligned}
& A_{n}=N_{n} \sin \pi a t_{\mathrm{OTP}} \frac{n}{b}+K_{n} \frac{b}{\pi n a} \cos \pi a t_{\mathrm{OTP}} \frac{n}{b}, \\
& B_{n}=N_{n} \cos \pi a t_{\mathrm{OTP}} \frac{n}{b}-K_{n} \frac{b}{\pi n a} \sin \pi a t_{\mathrm{OTP}} \frac{n}{b},
\end{aligned}
$$

where

$$
N_{n}=\frac{2}{b} \int_{0}^{b} u\left(x, t_{\text {OTP }}\right) \sin \pi x \frac{n}{b} d x, \quad K_{n}=\frac{2}{b} \int_{0}^{b} u_{t}\left(x, t_{\text {OTP }}\right) \sin \pi x \frac{n}{b} d x .
$$

Thus, if separation of load and blade will take place, the separate movement of a blade will be described by function

$$
v(x, t)=A\left(\sin \frac{\omega}{a} x+\sin \frac{\omega}{a}(b-x)\right) \frac{1}{\sin \omega b / a}+\sum_{n=1}^{\infty}\left(A_{n} \sin \pi a t \frac{n}{b}+B_{n} \cos \pi a t \frac{n}{b}\right) \sin \pi x \frac{n}{b},
$$

In a case of $n$ strips, the problem becomes complicated as separation can take place only in parts of strips, and in this case movement of a blade and part of strips, which has stayed on it, will be described by an equation of the kind (2.1). In this case, problem about movement of a blade is necessary to solve in principle the same way as in case of movement of system without separation. However in each concrete case, the various layers of load can separate from blade and in various sequences, therefore problem becomes multivariant.

\section{Connection of the load and the blade}

Further there will be a connection of a load and a blade at the moment of time $t_{c \mu}$, which is determined as the least positive root of the equation $z(t)=v\left(h_{i}, t\right)$, greater than $t_{\text {OTP }}$. At $t>t_{c \mu}$, the system again will change structure, and at this stage again it is necessary to consider a problem about joint movement of a blade and a load. This problem is similar to the one considered at the beginning, only initial conditions in case of two stripes will look as

$$
u\left(x, t_{c \mu}\right)=v\left(x, t_{c \mu}\right), \quad u_{t}\left(x, t_{c \mu}\right)=\psi_{2}(x),
$$

where the function $\psi_{2}(x)$ is under construction, proceeding from the following reasons. The blade at $t=t_{c \mu}$ has speed $v_{t}\left(x, t_{c \mu}\right)$. Besides, on straight lines $x=h$ and $x=b-h$ on a blade, at this moment the load having speed $z^{\prime}\left(t_{c \mu}\right)$ falls. Proceeding from preservation of quantity of movement on straight lines $x=h$ and $x=b-h$, we accept that at the moment of time $t=t_{c \mu}$ the speed on these straight lines is

$$
v_{h}=\frac{\rho v_{t}\left(x, t_{c \mu}\right)+\rho_{H} z^{\prime}\left(t_{c \mu}\right)}{\rho+\rho_{H}} .
$$

Therefore $\psi_{2}(x)$ is equal to $v_{t}\left(x, t_{c \mu}\right)$, if $x \neq h$ or $x \neq b-h$, and is equal $v_{h}$, if $x=h$ or $x=b-h$. 


\section{Connected movement of load and blade}

The solution of the problem on the connected movement of load and blade after their joining, that is at $t>t_{c \mu}$, is found as the sum of three functions:

$$
u(x, y, t)=u_{1}(x, y, t)+u_{31}(x, y, t)+u_{4}(x, y, t) .
$$

Here functions $u_{1}(x, y, t)$ and $u_{31}(x, y, t)$ are the same ones that have been obtained above with formulas (3.2) and (3.55) accordingly. We only recall that finally function $u_{1}(x, y, t)$ is obtained as

$$
u_{1}(x, y, t)=X(x) \sin \omega t
$$

where $X(x)$ is determined by formula (3.30). Function $u_{4}(x, y, t)$ is similar to the sum of functions $u_{2}(x, y, t)$ and $u_{32}(x, y, t)$, is the solution of homogeneous equation appropriate (2.1), and satisfies the boundary conditions (2.2) and (2.3) and the initial conditions

$$
\begin{aligned}
u_{4}\left(x, y, t_{c \mu}\right) & =v\left(x, t_{c \mu}\right)-u_{1}\left(x, t_{c \mu}\right)-u_{31}(x), \\
u_{4, t}\left(x, y, t_{c \mu}\right) & =\psi_{2}(x)-u_{1, t}\left(x, t_{c \mu}\right) .
\end{aligned}
$$

The form of solution $u_{4}$ is represented by formula (3.49). However, owing to that, as discovered above, solution of the considered problem is independent from variable $y$, hence in formula (3.49) we have to put $n=0$, and therefore form of solution becomes more simply:

$$
u_{4}(x, t)=\sum_{m=1}^{\infty} X_{0 m}(x)\left(A_{0 m} \sin \frac{a}{b} z_{0 m} t+B_{0 m} \cos \frac{a}{b} z_{0 m} t\right) .
$$

Having designated

$$
\begin{aligned}
& \varphi(x)=v\left(x, t_{c \mu}\right)-u_{1}\left(x, t_{c \mu}\right)-u_{31}(x), \\
& \psi(x)=\psi_{2}(x)-u_{1, t}\left(x, t_{c \mu}\right),
\end{aligned}
$$

we build expansion of these functions of the kind

$$
\varphi(x)=\sum_{m=1}^{\infty} b_{0 m} X_{0 m}(x), \quad \psi(x)=\sum_{m=1}^{\infty} c_{0 m} X_{0 m}(x),
$$

where

$$
\begin{aligned}
X_{0 m}(x)= & \sin \frac{z_{0 m} x}{b}-\frac{a^{2} \rho_{H} z_{0 m}}{T b} \sin \frac{z_{0 m}}{b} h \sin \frac{z_{0 m}}{b}(x-h) H(x-h) \\
& -\frac{a^{2} \rho_{H} z_{0 m}}{T b}\left[\sin \frac{z_{0 m}}{b}(b-h)-\frac{a^{2} \rho_{H} z_{0 m}}{T b} \sin \frac{z_{0 m}}{b} h \sin \frac{z_{0 m}}{b}(b-2 h)\right] \\
& \times \sin \frac{z_{0 m}}{b}(x-(b-h)) H(x-(b-h)) .
\end{aligned}
$$


Thus coefficients of expansion of initial functions have to be determined from system of equations

$$
\begin{gathered}
\sum_{m=1}^{\infty} b_{0 m} \int_{0}^{b} X_{0 m}(x) X_{0 s}(x) d x=\int_{0}^{b} \varphi(x) X_{0 m}(x) d x \\
\sum_{m=1}^{\infty} c_{0 m} \int_{0}^{b} X_{0 m}(x) X_{0 s}(x) d x=\int_{0}^{b} \psi(x) X_{0 m}(x) d x, \quad s=1,2, \ldots
\end{gathered}
$$

After that, having substituted the form of the solution (6.4) in the initial conditions (6.3) in view of expansion (6.6) and having equated factors in the left and right parts of the turned-out equalities at $X_{0 m}(x)$ with identical values $m$, we will receive systems of the equations

$$
\begin{aligned}
& A_{0 m} \sin \frac{a}{b} z_{0 m} t_{c \mu}+B_{0 m} \cos \frac{a}{b} z_{0 m} t_{c \mu}=b_{0 m} \\
& A_{0 m} \cos \frac{a}{b} z_{0 m} t_{c \mu}-B_{0 m} \sin \frac{a}{b} z_{0 m} t_{c \mu}=\frac{b c_{0 m}}{a z_{0 m}}
\end{aligned}
$$

$m=1,2, \ldots$, whence

$$
\begin{aligned}
& A_{0 m}=b_{0 m} \sin \frac{a}{b} z_{0 m} t_{c \mu}+\frac{b c_{0 m}}{a z_{0 m}} \cos \frac{a}{b} z_{0 m} t_{c \mu} \\
& B_{0 m}=b_{0 m} \cos \frac{a}{b} z_{0 m} t_{c \mu}-\frac{b c_{0 m}}{a z_{0 m}} \sin \frac{a}{b} z_{0 m} t_{c \mu} .
\end{aligned}
$$

\section{Further movements}

As at $t>t_{c \mu}$ separation of a load from a blade also can take place, the necessary and sufficient separation conditions (4.1) again are considered, but already with function $u(x, t)$, being the solution of initial-boundary value problem (2.1), (2.2), (2.3), (5.1). The least positive root $t_{\mathrm{OTP} 1}$ of $(4.1)$, responding to a condition $t_{\mathrm{OTP} 1}>t_{c \mu}$, is considered as the moment of possible repeated separation of a load from a blade. Then, under formula (4.5) with replacement in it of function $u(x, t)$ by the solution of a problem (2.1), (2.2), (2.3), (5.1) and $t_{\text {OTP }}$ by $t_{\text {OTP } 1}$, separate movement of a load is determined. Thus under formula (4.19), in which the factors $A_{n}$ and $B_{n}$ are still calculated under formula (4.17), but the factors $N_{n}$ and $K_{n}$ should be determined for functions $\varphi_{1}$ and $\psi_{1}$, looking like (4.14),

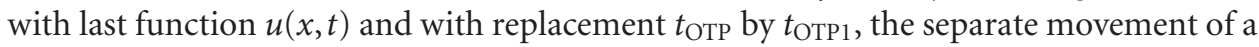
blade is determined. After that the sufficient condition of separation, inequality (4.1) for $t>t_{\mathrm{OTP} 1}$ is checked. If this condition is satisfied, that is, separation has taken place, again from the equation $z(t)=v\left(h_{i}, t\right)$ the moment of coupling of a load with a blade $t_{c \mu 1}$ as the least positive root of this equation, greater than $t_{\mathrm{OTP} 1}$, is determined. Then at $t>t_{c \mu 1}$, a problem such as (2.1), (2.2), (2.3), (5.1), describing joint movement of a load and a blade up to next separation, is solved. And so on. To consider some final quantity of cycles both separating and coupling a load and a blade it is necessary, as from follows the formulas of solutions, that the functions describing movement of a load and a blade be not periodic.

The separate interest represents search of periodic modes of movement of such system [2]. 
410 Interaction of vibrating surfaces with materials

\section{References}

[1] V. V. Leljakova and V. A. Ostapenko, The generalized general solution of one class of the differential equations, The Bulletin DSU. Mathematics. Dnepropetrovsk. Issue 2, 1996.

[2] V. A. Ostapenko, Necessary and sufficient conditions of existence of the periodic solutions in systems with variable structure, The Bulletin DNU. Mathematics. Dnepropetrovsk. Issue 7, 2002.

[3] Cross oscillations of a membrane with the large loadings, concentrated on lines, The Differential equations and their applications. -D. (1999).

[4] A. N. Tihonov and A. A. Samarskiy, The equations of mathematical physics, Science, M. (1966), -C. 724 (Russian).

V. A. Ostapenko: Faculty of Mathematics and Mechanics, National University of Dnepropetrovsk, 13 Nauchnaya Street, 49050 Dnepropetrovsk, Ukraine

E-mail address: mmf@ff.dsu.dp.ua 


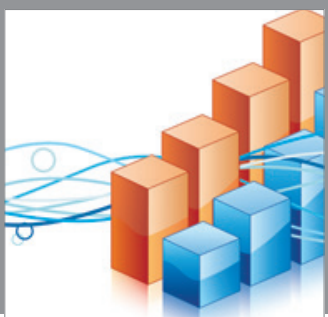

Advances in

Operations Research

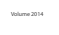

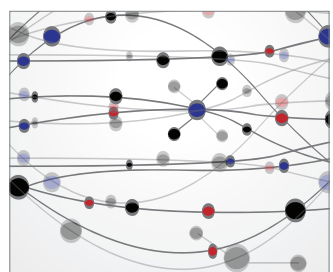

\section{The Scientific} World Journal
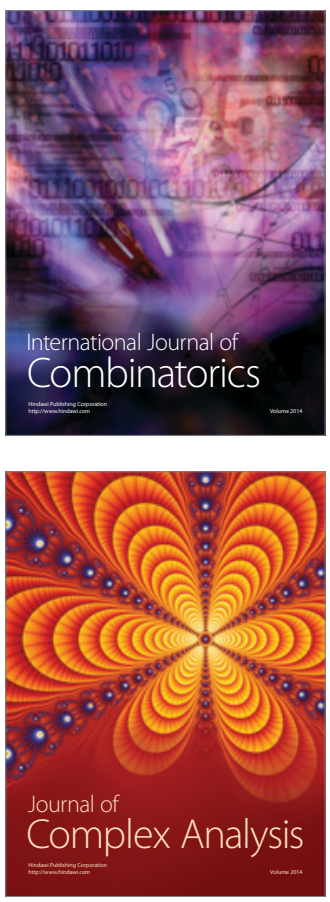

International Journal of

Mathematics and

Mathematical

Sciences
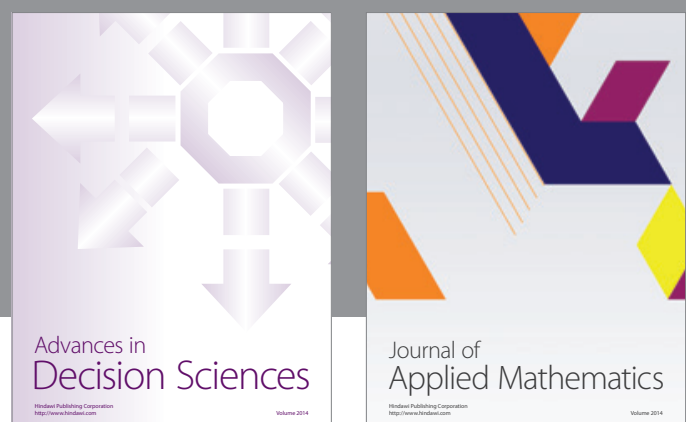

Journal of

Applied Mathematics
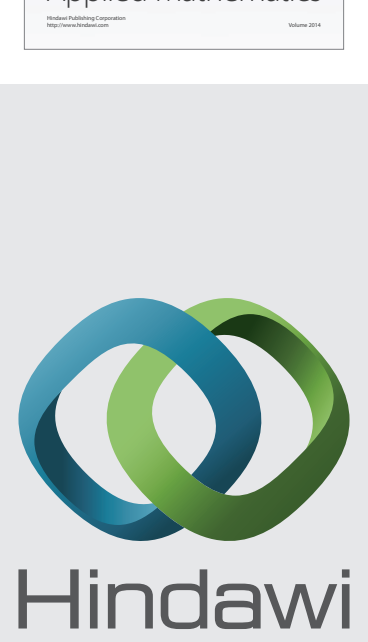

Submit your manuscripts at http://www.hindawi.com
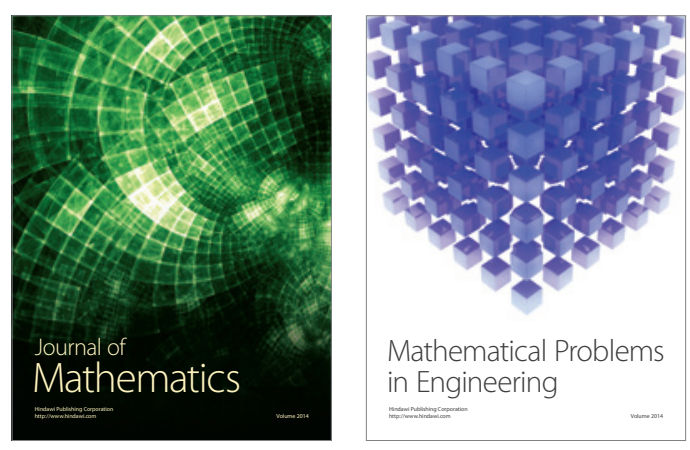

Mathematical Problems in Engineering
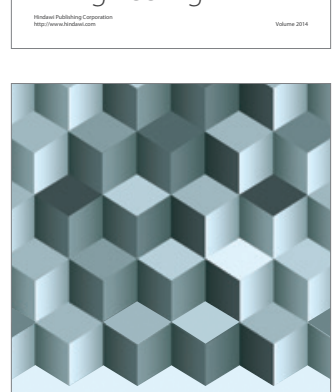

Journal of

Function Spaces
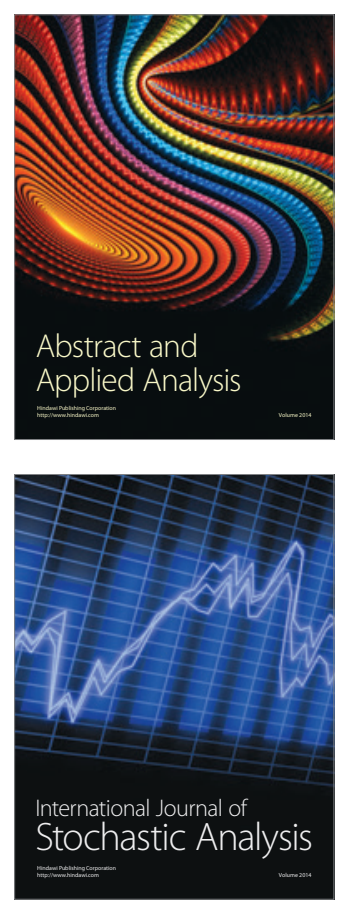

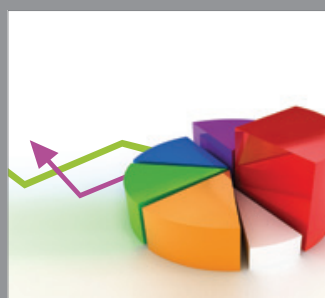

ournal of

Probability and Statistics

Promensencen
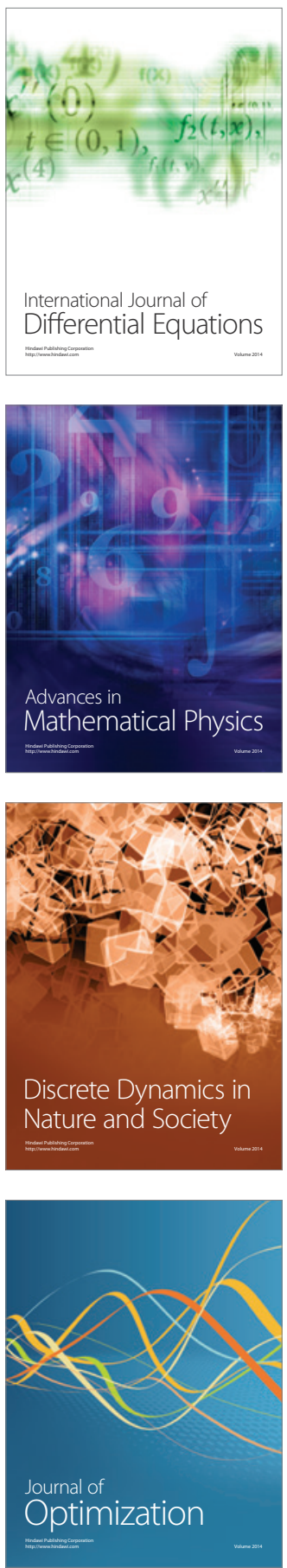